\title{
Optimistic Atomic Multicast
}

\author{
Carlos Eduardo Bezerra* ${ }^{*}$, Fernando Pedone* ${ }^{*}$, Benoît Garbinato $^{\dagger}$, Cláudio Geyer $^{\ddagger}$ \\ ${ }^{*}$ University of Lugano, ${ }^{\dagger}$ University of Lausanne, ${ }^{\ddagger}$ Universidade Federal do Rio Grande do Sul
}

\begin{abstract}
Message ordering is one of the cornerstones of reliable distributed systems. However, some ordering guarantees, such as atomic order, are expensive to implement in terms of message delays. This paper presents Optimistic Atomic Multicast, a protocol that combines reduced latency and increased throughput. Messages can be delivered optimistically in a single communication step and conservatively in three communication steps. Differently from previous optimistic group communication protocols, Optimistic Atomic Multicast does not rely on spontaneous message ordering for fast delivery. In addition to presenting Optimistic Atomic Multicast, we provide detailed performance results comparing it to other ordering protocols in both local-area and wide-area networks.
\end{abstract}

Keywords-fifo reliable multicast; atomic multicast; quasigenuine; optimistic delivery

\section{INTRODUCTION}

Message ordering is one of the cornerstones of reliable distributed systems and lies at the core of fundamental faulttolerant approaches such as state-machine replication [1], [2]. In state-machine replication, servers are replicated so that the failure of one or more replicas does not prevent client requests from being executed against non-faulty replicas. State-machine replication requires that (i) every correct replica receive all requests and (ii) no two replicas disagree on the order of received requests. These requirements are captured by group communication primitives that ensure total order, such as atomic broadcast [3].

Atomic broadcast simplifies the design of reliable distributed systems. However, it is typically expensive to implement (e.g., in terms of communication steps) and does not scale well (i.e., with the number of nodes involved in the ordering protocol). The first shortcoming is particularly problematic if the application is geographically distributed since it has been shown that atomic broadcast requires three communication steps [4]. The second shortcoming implies that throughput, measured in number of requests ordered by time unit, cannot be increased by adding nodes to the system, an inherent limitation of total order.

The distributed systems research community has been long aware of these problems and solutions to each one of them have been proposed. Atomic broadcast protocols with reduced latency have been designed based on optimistic assumptions about spontaneous message ordering (e.g., [5], [6], [7]), relaxed ordering requirements, dependent on application semantics (e.g., [8]), or both (e.g., [9], [10]). Atomic multicast addresses the (lack of) scalability of atomic broadcast. While atomic broadcast propagates messages to all system members, atomic multicast divides the members into groups and propagates messages to some groups only, not all. If the atomic multicast protocol is genuine [11], then only the message's addressees (i.e., a set of groups) and sender will coordinate to ensure order. As a result, atomic multicast can scale better than atomic broadcast. This is beneficial for applications whose state can be decomposed into sub-states, each one associated to a group (e.g., [12]) since throughput can be increased by multicasting requests to sets of groups, not the whole system.

This paper presents Optimistic Atomic Multicast, the first atomic multicast protocol to exploit optimism, and thus, to combine reduced latency and increased throughput. Messages can be delivered optimistically in a single communication step and conservatively in three communication steps. To deliver messages optimistically, a process estimates a wait window based on expected message transmission delays and skews between node clocks. If the process' estimate holds, the order in which messages are delivered optimistically (i.e., the optimistic order) will match the conservative order.

Optimistic Atomic Multicast is quasi-genuine: not only the message's sender and destinations coordinate to establish order, but other groups may participate too. The set of groups that participate in the ordering of a message is determined by a relation, defined by the application. Moreover, this relation may change over time, a reconfiguration, so groups can be added and removed on-the-fly, reflecting the application needs. Reconfigurations are expected to be used sparingly though since they incur additional communication.

The contributions of this paper are: (1) An atomic multicast protocol that delivers messages in three communication delays. (2) A variation of this protocol that improves latency and delivers messages optimistically in a single communication delay. (3) A detailed experimental assessment of these protocols both in local-area and wide-area networks.

The remainder of the paper is organized as follows. Section II presents the system model and some definitions. Section III introduces Baseline Atomic Multicast. Section IV contains the optimistic version of the algorithm. Section V describes reconfiguration. Section VI presents our prototype and Section VII evaluates its performance. Section VIII reviews related works. Section IX concludes the paper. A proof of correctness of our protocols is available in a technical report [13]. 


\section{SYSTEM MODEL AND DEFINITIONS}

In the following, we introduce the underlying system model (Section II-A) and define the consensus abstraction (Section II-B), atop of which we have designed our atomic multicast protocols.

\section{A. Processes and communication}

We consider a system $\Pi=\left\{p_{1}, \ldots, p_{n}\right\}$ of processes that communicate through message passing and do not have access to a shared memory or a global clock. The system is asynchronous: messages may experience arbitrarily large (although finite) delays and there is no bound on relative process speeds. We assume the benign crash-stop failure model: processes may fail by crashing, but do not behave maliciously. A process that does not crash is correct; otherwise it is faulty. We define $\Gamma=\left\{g_{1}, \ldots, g_{m}\right\}$ as the set of process groups in the system. Groups are disjoint, nonempty, and satisfy $\bigcup_{g \in \Gamma} g=\Pi$. For brevity, we write " $p \in \gamma$ " instead of " $\exists g \in \gamma: p \in g$ ", where $\gamma$ is any set of groups such that $\gamma \subseteq \Gamma$.

Processes can also communicate using fifo reliable multicast, defined by the primitives fr-mcast $(\gamma, m)$ and fr-deliver $(m)$, where $m$ is a message and $\gamma$ is the set of groups $m$ is addressed to. Message $m$ has two fields: $m . s r c$, $m$ 's source group, and m.data, the application-specific content. Fifo reliable multicast guarantees that (i) if a correct process $p$ fr-mcasts $m$, then eventually all correct processes $q \in \gamma$ fr-deliver $m$ (validity); (ii) if a correct process $p$ fr-delivers $m$, then eventually every correct process $q \in \gamma$ fr-delivers $m$ (agreement); (iii) for any process $p$ and any message $m, p$ fr-delivers $m$ at most once, and only if $p \in \gamma$ and $m$ was previously fr-mcast (uniform integrity); and (iv) if $p$ fr-mcasts $m$ to $\gamma$ and then $m^{\prime}$ to $\gamma^{\prime}$, then no $q \in \gamma \cap \gamma^{\prime}$ fr-delivers $m^{\prime}$ without first fr-delivering $m$ (fifo order).

We say that a group $g$ executes the actions of sending, receiving, multicasting and delivering a message with the meaning that one or more processes in $g$ execute the action.

\section{B. Consensus}

An important part of this work relies on consensus to ensure that processes agree upon which messages are delivered and in which order they are delivered. Thus, we assume that consensus can be solved within a group. Moreover, we distinguish multiple instances of consensus executed within the same group with unique natural numbers. Consensus is defined by the primitives propose pro $_{g}(k, v)$ and decide $g(k, v)$, where $g$ is a group, $k$ a natural number and $v$ a value. Consensus satisfies the following properties in each instance $k$ : (i) if process $p \in g$ decides $v$, then $v$ was previously proposed by some process in $g$ (uniform integrity); (ii) if $p \in g$ decides $v$, then all correct processes in $g$ eventually decide $v$ (uniform agreement); and (iii) every correct process in $g$ eventually decides exactly one value (termination).

\section{Baseline Atomic Multicast}

In this section we define the atomic multicast problem (Section III-A), introduce the Baseline Atomic Multicast algorithm (Sections III-B and III-C) and discuss its liveness guarantees (Section III-D).

\section{A. Problem definition}

Atomic multicast allows messages to be sent to a set $\gamma$ of groups. It is defined by the primitives multicast $(\gamma, m)$ and deliver $(m)$, where $m$ is a message with fields $m$.src and m.data, as defined in Section II-A. Atomic multicast guarantees the following properties:

(i) If a correct process $p$ multicasts $m$, then every correct process $q \in \gamma$ delivers $m$ (validity).

(ii) If $p$ delivers $m$, then every correct process $q \in \gamma$ delivers $m$ (uniform agreement).

(iii) For any message $m$, every process $p \in \gamma$ delivers $m$ at most once, and only if some process has multicast $m$ previously (uniform integrity).

(iv) If $p$ multicasts $m$ and then $m^{\prime}$ to groups $\gamma$ and $\gamma^{\prime}$, respectively, then no process $q$ in both $\gamma$ and $\gamma^{\prime}$ delivers $m^{\prime}$ before delivering $m$ (fifo order).

(v) No two processes $p$ and $q$ in both $\gamma$ and $\gamma^{\prime}$ deliver $m$ and $m^{\prime}$ in different orders (atomic order).

Atomic multicast generalizes atomic broadcast, where every message is multicast to all groups. To implement atomic multicast using an atomic broadcast algorithm, it suffices to broadcast every message $m$ to all groups, and those not interested in $m$ discard it. Obviously, this is not efficient. To rule out this kind of implementation, an early work introduced the notion of genuine atomic multicast algorithms [11], which spare unnecessary communication: to deliver message $m$ multicast to $\gamma$, groups $g$ and $h$ only communicate if they are "concerned by $m$ ". Group $x$ is concerned by $m$ if the sender of $m$ is in $x$ or $x \in \gamma$. While genuineness is an important property for atomic multicast protocols, it is expensive: no genuine multicast protocol can deliver messages in fewer than two inter-group network delays [14]. Since we seek communication-efficient algorithms, we introduce next the concept of quasi-genuine atomic multicast protocols.

In a quasi-genuine atomic multicast protocol, the groups that communicate to deliver a message $m$ are determined by an a priori sendersTo relation, defined based on the application semantics and access patterns-as opposed to $m$ 's sender and destination groups. For any group $g$, we define sendersTo $(g)$ as the set of groups that can multicast a message to $g$. If $m$ is multicast to a set of groups $\gamma$, then all groups in sendersTo $(g)$, where $g \in \gamma$, can communicate to deliver $m$, even if some group $h$ in $\operatorname{sendersTo}(g)$ is neither $m$ 's sender nor one of $m$ 's destination groups. We denote the sendersTo relation the system's configuration. A sendersTo relation can be changed via a reconfiguration, described in Section V. 
Applications whose data can be partitioned according to access locality can benefit from a quasi-genuine protocol. Consider a database divided in partitions $P_{1}$ and $P_{2}$ such that some data items are exclusively in $P_{1}$, some are exclusively in $P_{2}$, and some items are shared by both partitions-in other words, the partitions are not disjoint. Most commands access (i.e., read and write) items in one partition only and few commands include shared items. In this context, we create groups $g_{1}, g_{2}$ and $g_{3}$ such that processes in $g_{1}$ and $g_{2}$ replicate exclusive items in $P_{1}$ and $P_{2}$, respectively, and processes in $g_{3}$ replicate items shared by $P_{1}$ and $P_{2}$. A command $C$ that accesses items in partition $P_{x}$, where $x \in\{1,2\}$, is handled by a process $p$ in group $g_{x}$. If $C$ accesses items exclusive to $P_{x}$ only, then $p$ multicasts $C$ to $g_{x}$; if $C$ includes exclusive and shared items in $P_{x}, p$ multicasts $C$ to $g_{x}$ and $g_{3}$; finally, if $C$ accesses only shared items, $p$ multicasts $C$ to $g_{3}$. Consequently, sendersTo $\left(g_{1}\right)=\left\{g_{1}\right\}$, sendersTo $\left(g_{2}\right)=\left\{g_{2}\right\}$ and sendersTo $\left(g_{3}\right)=\left\{g_{1}, g_{2}\right\}$. This scheme allows commands that access items stored exclusively in $P_{1}$ to be ordered independently of commands that access items stored exclusively in $P_{2}$.

\section{B. Overview of the algorithm}

Hereafter, we assume that each message $m$ has two additional fields, m.ts, the message's timestamp, and m.dst, the set of groups $m$ is addressed to. To multicast $m$ to $\gamma$, process $p$ in group $g$ sets $m$.ts to a unique initial timestamp and then fr-mcasts $m$ to all processes in $g$. Such timestamp is created based on $p$ 's wallclock (i.e., $p$ 's real-time local clock) and $p$ 's unique identifier.

When processes in $g$ fr-deliver $m$, they run a consensus instance within $g$ to agree on $m$ 's final timestamp, after possibly adjusting it to ensure the following invariant: for any two messages $m$ and $m^{\prime}$, multicast by processes in $g$, if a process in $g$ decides $m$ before $m^{\prime}$, then $m$.ts $<m^{\prime}$.ts. This is important since we intend to deliver messages according to their final timestamp order. The initial timestamp assigned by the message's sender may violate the invariant due to the asynchrony of the system. If so, processes reassign the message's timestamp after consensus to keep the invariant.

Once group $g$ has decided on $m$ 's final timestamp, $m$ is fr-mcast to all its destination groups $\gamma$. Let $h$ be a group in $\gamma$. To ensure that no message $m^{\prime}$ with a smaller final timestamp than $m$ 's (i.e., $m^{\prime} . t s<m . t s$ ) is delivered after $m$, process $q \in h$ delivers $m$ only when it has received at least one message from every group $x$ in sendersTo $(h)$ with a timestamp greater than $m$ 's timestamp. We denote each such a message barrier $(x)$.

More precisely, a barrier is the guarantee that a group $x$ gives to some other group $h$ that $x$ will not send to $h$ any new messages with a timestamp lower than barrier $(x)$. As all groups receive messages from each other in the order of their timestamps, ensured by fr-mcast, every message exchanged between groups is a barrier.

\section{Detailed description}

To multicast $m, p$ assigns $m$ 's initial timestamp and fr-mcasts $m$ to the other processes in $g$ (lines 7-10 in Algorithm 1). Primitive getTime() (line 9) returns a unique value based on the process' unique identifier and current wallclock value. After fr-delivering $m$, each process in $g$ puts $m$ in messages (line 13) to be proposed until it is decided (lines 19-20), along with other undecided messages. As all correct processes in $g$ do so, some decision will eventually contain $m$.

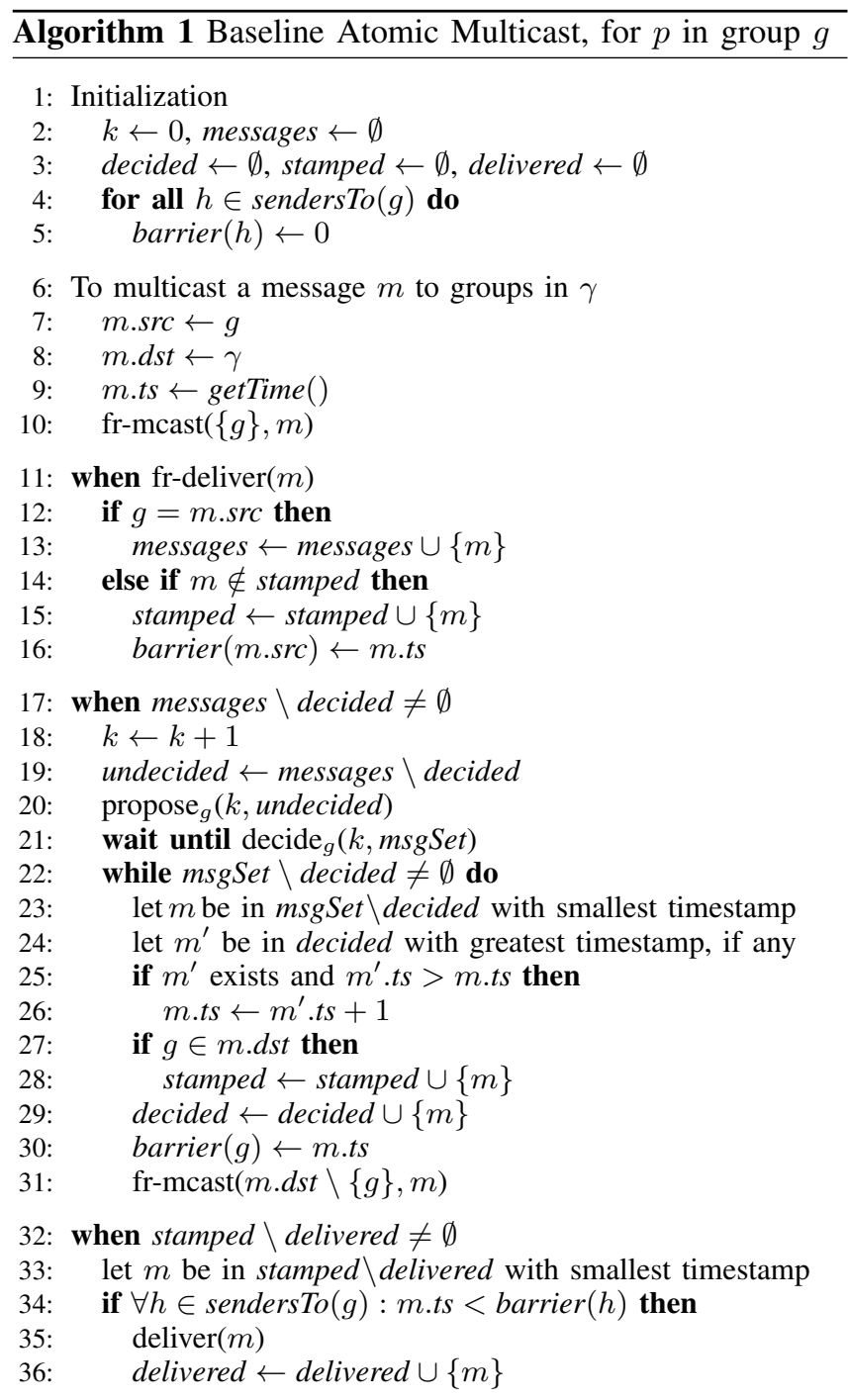

Algorithm variables:

messages: messages multicast by processes in $g$

decided: messages proposed and decided within $g$

stamped: messages with a final timestamp sent to $g$ by any group; to be delivered, some messages may need barriers from groups in sendersTo $(g)$

delivered: messages already delivered by $p$ 
When a set of messages is decided, such messages are handled by each process in $g$ in ascending order of the initial timestamps (line 23). If $m$ has a timestamp that is lower than that of some previously decided message $m^{\prime}$ (lines 24-25), then $m . t s$ is changed to a value greater than $m^{\prime} . t s$ (line 26). Doing so will ensure that all messages from $g$ are delivered in the order of their final timestamps.

After deciding $m, p$ checks whether $g$ is one of its destinations (line 27). If so, $m$ is inserted into stamped (line 28 ), meaning that $p$ should deliver it eventually. Then, $m$ is included into decided (line 29) so that $p$ stops proposing $m$ in the following consensus instances. As no message decided afterwards within $g$ will have a timestamp lower than $m$ 's, $p$ sets barrier $(g)$ to m.ts (line 30 ). Then, if $m$ has any destination other than $g$, say $h, p$ fr-mcasts $m$ to $h$ (line 31). When some process $q$ in $h$ fr-delivers $m$ for the first time (lines 11 and 14), $q$ inserts it into stamped (line 15) and sets barrier $(g)$ to $m$.ts (line 16). This is done since any previous message from $g$ has already been received.

Let $r$ be a process in some group $h^{\prime} \in$ m.dst. When (i) the stamped set at $r$ contains $m$, (ii) $m$ has the lowest timestamp among all undelivered messages in such set (lines 32 and 33), and (iii) $r$ has already received a barrier from every group in sendersTo $\left(h^{\prime}\right)$ with value greater than $m . t s$ (line 34), $r$ delivers $m$ (lines 35 and 36).

\section{Liveness guarantees}

Assume that group $g$ multicasts $m$ to group $h$. In Algorithm 1 , for process $q$ in $h$ to deliver $m, q$ must receive a barrier $b>m$.ts from every group $k$ in sendersTo $(h)$. Since the exchange of barriers among processes is triggered by the multicast of application messages, the algorithm assumes that every group in sendersTo $(h)$ periodically multicasts an application message to $h$. This assumption can be weakened by having each group $x$ in sendersTo $(h)$ multicast a null message if $x$ does not have any application messages to multicast to $h$ for a certain timeout $\Delta$. Null messages are not delivered to the application, they only carry a barrier.

Multicasting null messages periodically is a simple solution, however it may introduce unnecessary delays. If $q$ is only waiting for $x$ 's barrier to deliver $m$ and $x$ has just sent barrier $b<m$.ts to $h$, then unless $x$ has an application message to multicast to $h, x$ will only send another barrier to $h$ after another timeout $\Delta$. As a consequence, the delivery of $m$ may be delayed by up to $\Delta$. Besides, there is no guarantee that the next barrier sent by $x$ to $h$ will be greater than $m$.ts. Another solution is for $g, m$ 's source group, to request barriers on behalf of $m$ 's destination group $h$. As soon as process $p$ in $g$ knows the final timestamp of $m, p$ requests a barrier to every $x$ in sendersTo $(h)$. When processes in $x$ receive a barrier request for $m$, they must run a consensus instance to agree on a barrier with a timestamp greater than $m$ 's timestamp. This technique lowers delivery latency at the cost of having more messages exchanged between groups.

\section{Optimistic Atomic Multicast}

In the following, we modify Baseline Atomic Multicast to introduce optimistic delivery. The resulting algorithm, Optimistic Atomic Multicast, can deliver messages optimistically after one communication step, at the risk of delivering some of them out order. In brief, the idea is for a process $p$ to optimistically deliver a message $m$ after $p$ fr-delivers $m$; to minimize out of order messages, processes may delay the optimistic delivery by a small time window. We first define the Optimistic Atomic Multicast problem (Section IV-A) and then present the new protocol in detail (Section IV-B).

\section{A. Problem definition}

Optimistic Atomic Multicast is defined by primitives $\operatorname{multicast}(\gamma, m)$, opt-deliver $(m)$ and deliver $(m)$. Hereafter, we refer to the last two primitives as optimistic and conservative delivery, respectively. Thus, Optimistic Atomic Multicast delivers messages twice, once optimistically and once conservatively, although optimistic delivery requires fewer communication steps than conservative delivery.

Conservative delivery guarantees the five properties of atomic multicast, defined in Section III-A. Optimistic delivery guarantees validity, uniform integrity, and fifo order of atomic multicast; it ensures non-uniform agreement and probabilistic atomic order (i.e., atomic order is guaranteed under certain assumptions, as defined next). ${ }^{1}$

Applications can exploit this abstraction by processing a message as soon as it is optimistically delivered. If the optimistic delivery order of messages does not match their conservative delivery order, the application may need to rollback the processing of these messages and re-execute them in the correct order (e.g., [15], [16]). However, rolling back the processing of out-of-order messages is not needed if their relative order does not matter to the application (e.g., one message modifies the state of variable $x$ and the other message reads variable $y$ ).

\section{B. Delivering messages optimistically}

In Optimistic Atomic Multicast, processes in the destination set of a message $m$ predict $m$ 's atomic order from $m$ 's initial timestamp after one communication step. This is done as follows. When a process $p$ in $g$ multicasts $m, p$ fr-mcasts $m$ to all groups in $m$.dst, not only its own group. When $q$ in $m . d s t$ fr-delivers $m, q$ optimistically delivers $m$ following the order given by $m$ 's initial timestamp.

In order for $m$ 's optimistic order to match its conservative order, three conditions are sufficient: (a) there are no message losses; (b) $m$ 's source group does not change $m$ 's initial timestamp; and (c) before a process in $m$ 's destination groups optimistically delivers $m$, the process receives all messages with initial timestamp smaller than $m$ 's.

\footnotetext{
${ }^{1}$ Since agreement of optimistic delivery is non-uniform, if the sender crashes while multicasting a message, some processes may deliver the message optimistically but never conservatively.
} 
Condition (a) can be approximated with reliable point-topoint communication (e.g., TCP). We satisfy conditions (b) and (c) probabilistically using the following scheme. Assume $p$ is a process in $m$ 's source group or in one of $m$ 's destination groups. To satisfy conditions (b) and (c), respectively, $p$ must wait "long enough" before proposing $m$ in a consensus instance and before optimistically delivering $m$.

The problem is how to define the length of $p$ 's wait window, denoted $w(p)$. As we consider an asynchronous system, it is impossible to determine $w(p)$ deterministically; instead, we optimistically assume that $p$ can estimate $w(p)$. Let $\delta(p, q)$ be the time a message takes to go from $q$ to $p$ and $\epsilon(p, q)$ the skew between the clocks of $p$ and $q .^{2}$

Wait window. For process $p$ in group $g$, we define $w(p)$ as $\max _{q \in \operatorname{sendersTo}(g)}(\delta(p, q)+\epsilon(p, q))$

If our optimistic assumption holds, that is, every process can accurately estimate its wait window, then the order of optimistic delivery matches the order of the conservative delivery. In more detail, the optimistic delivery works as follows: After an undecided message $m$ has been fr-delivered by $p, p$ waits until its wallclock has a value greater than $m . t s+w(p)$ and then $p$ opt-delivers $m$ and proposes $m$ in the next consensus instance. At this moment, if the optimistic assumption holds, all messages that could possibly have an initial timestamp lower than m.ts have already been fr-delivered by $p$. Thus, such messages will be optimistically delivered in the order of their initial timestamps.

If every process $q$ of $g$ has estimated $w(q)$ correctly, then all processes in $g$ will propose messages in the same order, that is, following the initial timestamps. Therefore, there will be no timestamp changes and the optimistic delivery will be correct. This happens because each process $q$ waits for $w(q)$ before proposing a message, and the messages waiting to be proposed are proposed in their initial timestamp order. Not changing timestamps allows for a further improvement: If barriers are requested for each message $m$, the barrier requests may be sent to the destination groups of $m$ at the same time as $m$ is fr-mcast by its sender. Such requests are also sent with a reliable multicast primitive, before the sender fr-mcasts $m$ in the first step of the algorithm.

If the optimistic assumption fails to hold and some message $m$ is received by some process $p$ after a message $m^{\prime}$ has been already opt-delivered by $p$, where $m . t s<m^{\prime} . t s$, then optimistic delivery violates atomic order. Out-of-order optimistic deliveries are detected by the application since they do not match the order of conservative of deliveries. The application must then handle these out-of-order messages if necessary.

\footnotetext{
${ }^{2}$ Notice that a negative skew means that the value of $q$ 's clock is higher than the value of $p$ 's clock, and so $p$ can wait less for messages from $q$, as they will have higher timestamps than $p$ 's messages.
}

\section{RECONFIGURATION}

A reconfiguration allows the sendersTo relation to change during the execution. Since including a process in the relation and removing a process from the relation follow similar procedures, we focus next on inclusion. For process $p$ in group $g$ to multicast a message to group $h, p$ creates a reconfiguration request $m_{r q}$, where $m_{r q} . d s t=\{h\}$, timestamps $m_{r q}$ and fr-mcasts it to all processes in $g$. Upon fr-delivering $m_{r q}$, it is inserted into messages, eventually proposed and decided within $g$, and then reliably multicast to $h$. Once processes in $h$ receive $m_{r q}$, they enqueue a request response $m_{r p}$ to be proposed and eventually decided within $h$, where $m_{r p} . d s t=\{g\}$. As with multicast messages, both $m_{r q}$ and $m_{r p}$ may have their timestamps changed after consensus. The final timestamp of $m_{r p}$ defines the moment from which processes in $h$ will wait for barriers from $g$. No message $m$ with a timestamp greater than $m_{r p}$.ts will be delivered by the processes in $h$ until a barrier greater than m.ts is received from $g$. Once $m_{r p}$ is received by $g$, it can multicast messages to $h$. Such messages will have a timestamp greater than $m_{r p} . t s$, to ensure their correct delivery order.

Whenever a message $m$ is sent to $h$, its sender can request barriers to every group in $\operatorname{sendersTo}(h)$ so that $m$ can be delivered. For that, all groups in sendersTo $(h)$ must know one another. So, as soon as $g$ decides $m_{r q}$, it fr-mcasts a notification $m_{r n}$ to all groups in sendersTo $(h)$, containing the timestamp of the reconfiguration request. When each process $q \in \operatorname{sendersTo}(h)$ fr-delivers $m_{r n}, q$ knows that it has to send a barrier request to $g$ when sending messages to $h$ with a timestamp greater than $m_{r q} . t s$. This value is chosen because the reconfiguration request itself may be seen as a barrier from $g$ to $h$. Finally, $q$ checks among the history of messages from its group if there are messages addressed to $h$ with timestamps greater than $m_{r q}$.ts for which no barrier request has been sent to $g$. Then a barrier request is sent to $g$ regarding such messages. This is done to ensure progress when a reconfiguration takes place.

\section{IMPLEMENTATION}

In the following, we comment on the implementation of our prototype and assess the number of communication steps needed for the optimistic and the conservative deliveries. We base our analysis on "good runs", that is, those with no failures, no suspicions of failure, and no reconfigurations. In practice these are (hopefully) the most common cases. In the analysis, we assume a maximum network message delay $\delta$ and negligible processing time.

We implemented consensus using Paxos [17]. To ensure termination, Paxos assumes the existence of a leader. In our implementation, one process in each group is assigned the role of leader, coordinating the execution of consensus in its group. To propose a value, a process sends it to the leader of its group, which then proceeds with consensus. The leader optimizes latency by executing Phase 1 of Paxos 
before a value is proposed [17]. Consensus instances are run in parallel, one for each multicast message. Each group implements an independent instance of Paxos.

To determine $w(p)$, each process $p$ calculates an estimate delay plus clock skew for every process $q$ that sends messages to $p$. The value of $w(p)$ is the maximum among such estimates. Such an estimate, $p$ calculates the average of the difference between its current time and $m$ 's timestamp (i.e., getTime ()$-$ m.ts $)$ for the last 100 messages received from $q$ received by $p$. Notice that this difference accounts for both $\delta(p, q)$ and $\epsilon(p, q)$.

Consider a message $m$ multicast by $p$ in $g$ to the groups in $\gamma$. In order to optimistically deliver $m, p$ fr-mcasts $m$ to all groups in $\gamma$ and to all the processes in $g$. This step takes a delay of $\delta$. Instead of delivering $m$ optimistically and starting consensus as soon as $m$ is fr-delivered, processes in $g$ wait until their local time is greater than m.ts $+w(p)$. If process clocks are synchronized and message delay estimations are exact, then $w(p)$ will match $\delta$, and optimistic delivery and the start of consensus will happen with delay $\delta$. If clocks are not synchronized or message delay estimations are inaccurate, then $w(p)$ may be too large, and processes will wait longer than necessary to start consensus, or $w(p)$ may be too small, and consensus may start too early, before a message $m^{\prime}$ with timestamp smaller than $m$ 's is received, leading to the optimistic and conservative delivery of $m$ and $m^{\prime}$ to happen in different orders.

In the best case, consensus is executed in two communication steps (i.e., $2 \delta$ ), after which $m$ 's final timestamp is determined. Group $g$ must then fr-mcast $m$ to each group $h$ in $\gamma$. Since we implement consensus with Paxos and the destinations are learners, sending $m$ 's final timestamp to members of $h$ can be done in Paxos' Phase 2B messages, saving one communication step [17]. The optimization works as long as $g$ 's leader is not replaced; otherwise, more communication steps are needed [17]. Process $q$ will conservatively deliver $m$ when $q$ receives barriers with timestamp greater than $m$ 's final timestamp from every group $x$ in sendersTo $(h)$. The barriers necessary for delivering $m$ are requested by $p, m$ 's sender, right before fr-mcasting $m$ in the first step of the algorithm. At this time, $p$ only knows $m$ 's initial timestamp. Thus, processes in $x$ send a barrier to $q$ that is greater than $m$ 's initial timestamp. If $m$ 's initial and final timestamps are the same (i.e., the optimistic assumption holds), then $q$ will receive all its needed barriers three communication steps after $m$ is multicast: one step for the request to be transmitted from $p$ to $x$ plus two steps for consensus to be executed in $x$. In this case, the conservative delivery will have a latency of $3 \delta$. If $m$ 's initial and final timestamps differ, a new barrier request will be necessary. In a good run, the timestamp proposed by $g$ 's leader is decided, so $g$ 's leader can send the new barrier request to $x$ while running consensus in $g$, leading to a $4 \delta$ conservative latency.

\section{PERformance EVAluation}

We evaluated the proposed protocols in local-area (Section VII-A) and wide-area networks (Section VII-B). In all experiments, every process multicasts messages to other groups, at a constant rate, according to the sendersTo relation. Each run of an experiment took 150 seconds, and latency values are reported as their 95th percentile.

We compare our algorithms with Paxos amcast, a protocol we implemented for atomic multicast using Paxos, where a single set of acceptors act as a fault-tolerant sequencer for all messages multicast in the system, providing a global order for them.

\section{A. Local-area network experiments}

The local-area network experiments were executed in a cluster of HP SE1102 servers, each with two quad-core Intel Xeon L5420 processors and 8GB of main memory, running CentOS 6.264 bits. The servers were interconnected through an HP ProCurve2910al-48G Gigabit switch and their clocks were kept approximately synchronized by using NTP. There were five groups, each with three processes; processes were distributed among five different servers of the cluster.

In the following, we evaluate how our protocols behave with respect to different loads (Section VII-A1), different numbers of groups multicasting to each other (Section VII-A2), and different estimations of the wait window for the optimistic delivery (Section VII-A3).

1) Throughput, latency and mistakes versus load: Figure 1 shows how throughput and latency vary with the load, measured in number of messages multicast per process per second. The maximum throughput of each protocol is circled in the graph. We define maximum throughput as the throughput value that corresponds to the latency before its inflection point (also marked in the graph). Optimistic Atomic Multicast's maximum throughput is determined by the conservative delivery.

We can see that the maximum throughput of Optimistic Atomic Multicast (graph on the left) is lower than the baseline's. This is due to the higher overhead of Optimistic Atomic Multicast (i.e., the initial reliable multicast is sent to all destination groups, as opposed to the sender's group only). Paxos amcast has the lowest maximum throughput, since all messages are handled by the same set of acceptors.

The latency of optimistic delivery at the protocol's maximum throughput (graph on the right) is significantly lower than that of Paxos amcast and less than half that of baseline and conservative. The conservative delivery latency is higher than baseline's because, in the former, messages are proposed for consensus only after being opt-delivered, whereas in the latter they are proposed as soon as they are fr-delivered. Among the non-optimistic deliveries, Paxos amcast achieves the lowest latency since it does not have to send or wait for barriers to deliver messages. 

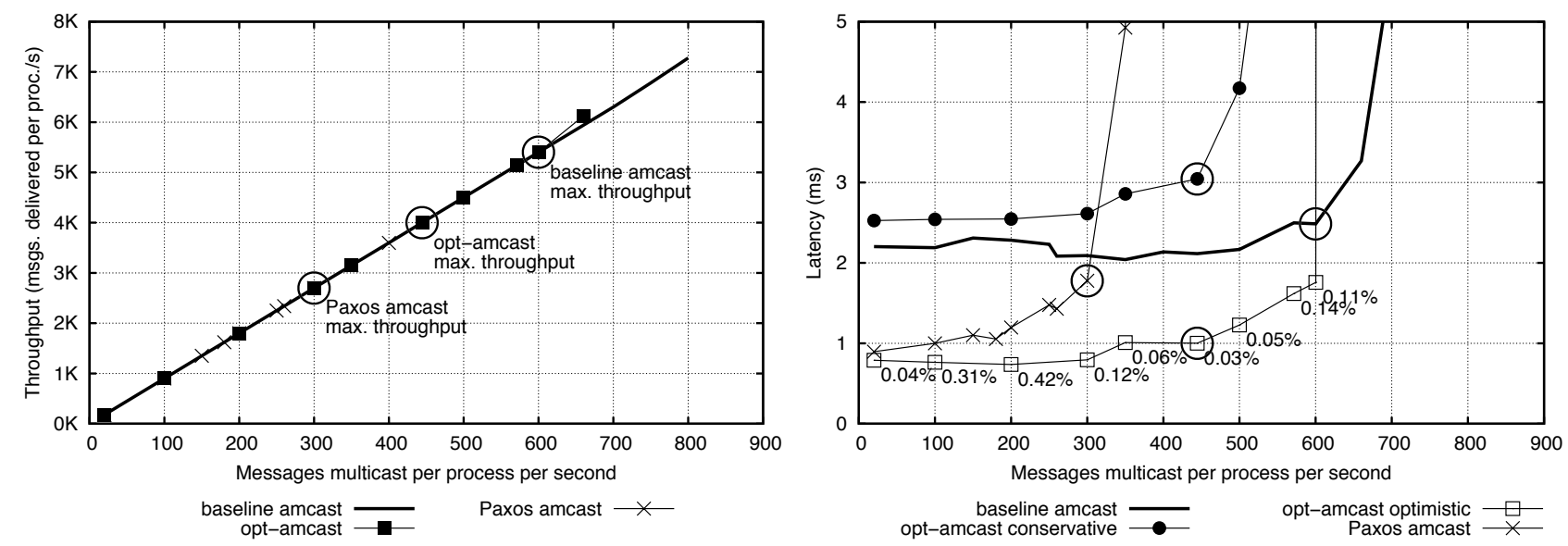

Figure 1. Throughput, latency and percentage of mistakes for $|\operatorname{sendersTo}(g)|=3$, for every $g$, in a local-area network.
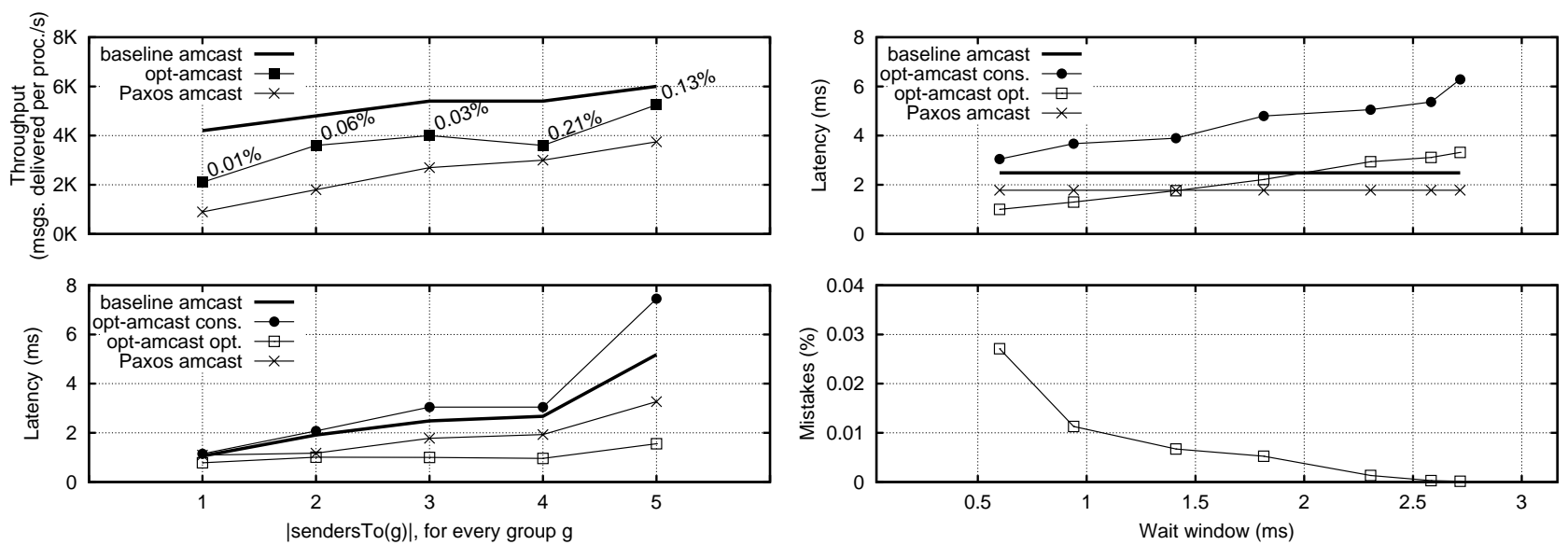

Figure 2. Impact of number of communicating groups and wait-window size on throughput and latency (percentages are mistakes in optimistic delivery).

Figure 1 (right) also shows the percentages of mistakes of the optimistic delivery, i.e., the percentage of messages whose optimistic order does not match the conservative order. By using NTP to synchronize clocks and estimating a wait window before opt-delivering messages, the mistakes rate remained below $0.5 \%$.

2) Impact of the number of communicating groups: From Figure 2 (top left), the maximum throughput of each protocol tends to increase with the number of destinations of each message. This happens because as the number of destinations increases, more groups will deliver a message decided by each consensus instance. Therefore, configurations with more destinations make better use of consensus executions. As the number of destinations for each message augments, the throughput difference between Paxos and the other algorithms decreases; when every message is addressed to every group, all acceptors notify all processes in the system, which is the normal case for Paxos amcast, but not for the baseline and Optimistic Atomic Multicast. Even in such situation, Paxos amcast has lower throughput, since it has only one set of acceptors ordering all messages, while the other protocols have one set of acceptors per each group, distributing the load.

Still in Figure 2 (bottom left), we can see that the number of communicating groups has an impact on the optimistic delivery latency. This happens because the optimistic delivery latency in each process $p$ is affected by the wait window $w(p)$, which is based on the worst latency among the processes sending to $p$; as more processes are included in the calculation of $w(p)$, the chances of getting a process with a higher estimated delay plus clock skew increase. A more significant effect can be noticed on the baseline and conservative latencies. This happens because not always all required barriers are received immediately when a message is enqueued for delivery. 

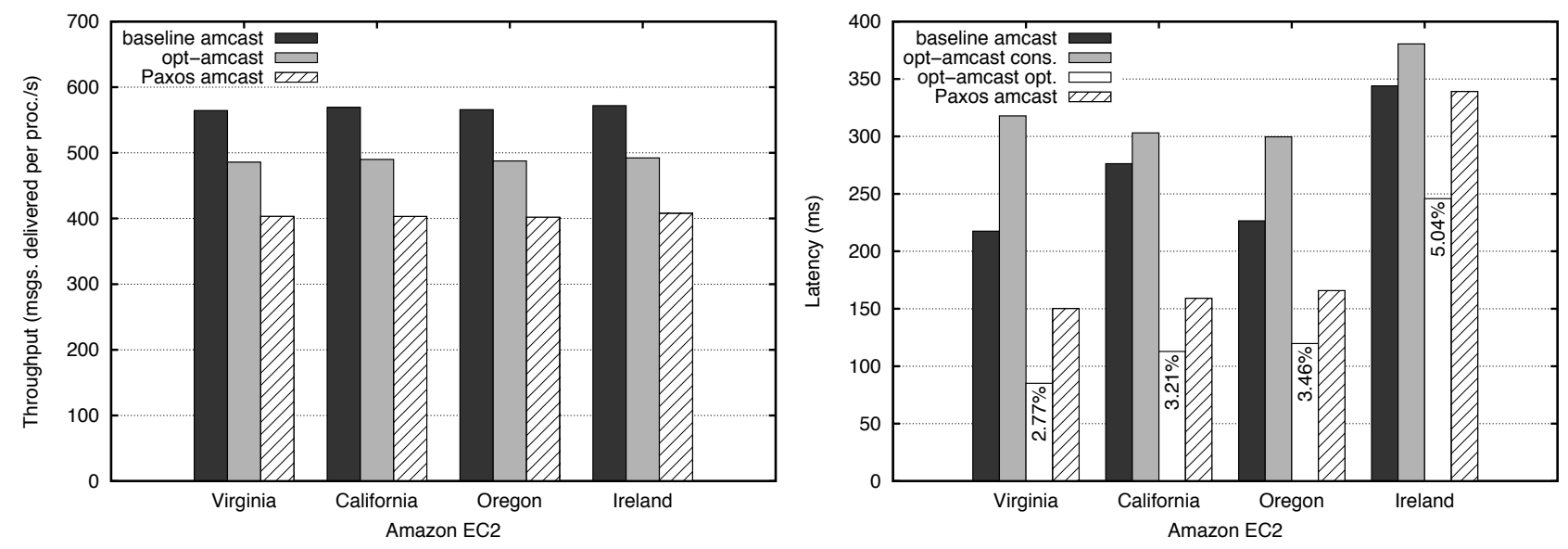

Figure 3. Throughput, latency and percentage of mistakes for $|\operatorname{sendersTo}(g)|=2$, for every $g$, in a wide-area network.

3) Impact of the estimated delay and clock skew: Figure 2 (top right) shows the latency and percentage of mistakes of the optimistic delivery on maximum throughput when each group can send messages to three other groups (i.e., $\forall g:|\operatorname{sendersTo}(g)|=3$ ). We can see that both the conservative and the optimistic delivery latencies increase with the length of the wait window, which depends on the estimated delay $\delta$ and clock skew $\epsilon$. The conservative delivery increases because a message is proposed only after it is opt-delivered, and so, the final delivery order tends to be the same as the optimistic one. As a reference, we show the latencies of the baseline protocol and Paxos amcast.

The percentage of mistakes decreases as the wait window becomes larger, as depicted in Figure 2 (bottom right). Waiting for more than $1 \mathrm{~ms}$ proved not to be very effective in comparison to how much it increased latency. Still, such small increase of the wait window (from $0.6 \mathrm{~ms}$ to $0.9 \mathrm{~ms}$, approximetely) was enough to reduce the mistakes rate by more than half, leading to an accuracy of over $99.98 \%$.

\section{B. Wide-area network experiments}

The wide-area experiments used four m1.large Amazon EC2 instances ${ }^{3}$ running Ubuntu Server 12.04.1 64 bits. Each instance was located in a different geographical region: three in the United States (Virginia, California and Oregon) and one in Ireland. To synchronize clocks, each of such instances queried a nearby public GPS-based NTP server, resulting in synchronization offsets below $10 \mathrm{~ms}$. It is worth noting that this deployment was fairly heterogeneous, since instances from different regions had different levels of processing power and network bandwidth. To report maximum throughput, we chose the load up to which latency was stable in every process. There were four multicast groups, each with four processes, one process from each geographical region.

\footnotetext{
${ }^{3}$ http://aws.amazon.com/ec2
}

In Figure 3, we can see the throughput and latency results in a WAN. As in the cluster, the maximum throughput achieved by baseline and Optimistic Atomic Multicast are fairly higher than that of Paxos amcast, with baseline's as the highest. Regarding latency, the optimistic delivery took the shortest time, in every region. We can see a high latency variation depending on the geographic location. The processes in Ireland experienced the highest latency, for every protocol evaluated. On the other extreme, the processes in Virginia had the lowest latencies for most protocols.

Finally, the rate of mistakes in the WAN experiments was much higher than in the cluster. It is a known fact that latencies in the Internet are much less predictable than in a local-area network, so a lower accuracy was not surprising. Still, we managed to achieve an accuracy around $95 \%$. This means that, even if all messages sent to a given destination require strict ordering among them, only 5\% might cause rollbacks. However, many applications can tolerate outof-order messages (e.g., two messages with requests that access different rows in the same partition). Therefore, when accounting for application semantics, 5\% of out-of-order messages will likely result in less than $5 \%$ of rollbacks.

\section{RELATED WORK}

There is a plethora of works in the literature related to atomic broadcast and multicast [18]. We review here those which are the most relevant to our work. To the best of our knowledge, no previous work presents all the features provided by our protocol, which is a quasi-genuine, reconfigurable, fault-tolerant, fifo atomic multicast that can deliver messages in three communication steps, with an optimistic delivery within one single step.

\section{A. Atomic broadcast and multicast}

Our atomic multicast protocols order messages using a mechanism that resembles Lamport's total order algo- 
rithm [1]: analogously to our barrier mechanism, a process considers an event to be the next in a total order of events when the process knows that such event has the lowest timestamp among all possible events to be considered in the system. Similarly to our protocols, Lamport's total order algorithm also relies on fifo, reliable channels. Differently than our protocols, it requires the participation of all processes in the system and does not tolerate failures.

One could see our barrier mechanism as a deterministic merge [19] algorithm. In particular, our protocols model each group as a merger and producer at the same time. To ensure that all mergers receive the same set of messages of all producers, we employ consensus and fifo reliable multicast. Finally, the merging is done by sorting all undelivered messages according to their timestamps and delivering those which have a timestamp up to the lowest of the last barriers received from the groups in sendersTo. Differently from [19], however, our protocols tolerate failures and do not require all mergers to receive all messages from all producers. Instead, each group may have a different sendersTo set. We also support reconfiguration, allowing the sendersTo relation to change over time.

Two fault-tolerant genuine atomic multicast algorithms are presented in [11]. Both are based on Skeen's multicast algorithm [20]. In the first algorithm, the destinations of each multicast message exchange timestamps for the message and, once each destination has received a timestamp from a majority of processes from each destination group, consensus is run among the destinations to decide the final timestamp. Upon decision, the message destinations coordinate to determine the message's position in the delivery queue. Such a protocol can deliver messages in $4 \delta$, assuming the best-case bound of $2 \delta$ for consensus. A variation of this algorithm [21], optimized for message size, has delivery latency of $5 \delta$.

The second algorithm in [11], similarly to [14] and [22], has an optimal delivery latency of two inter-group communication delays. Although based on Skeen's algorithm, they can tolerate failures by replacing each process by a group of processes. To deliver messages with low latency, communication within each group must be fast, so each group is contained in a single site (e.g., the same local-area network). As a result, these protocols are vulnerable to disasters, that is, the failure of the site. Although messages can be delivered with two inter-group delays, overall delivery latency (i.e., considering inter group and intra group communication) is $6 \delta$ in most cases and $4 \delta$ in a few special cases.

In [14], the authors propose a broadcast protocol which delivers messages in atomic order within one single communication step. This protocol is based on rounds: each process delivers the received messages in a round only after receiving the messages from all other groups for that round. To ensure liveness, groups need to constantly exchange messages, even if null. Rounds are conceptually similar to our barrier mechanism. Since our multicast protocols are quasi-genuine, not all groups have to coordinate to deliver messages. Furthermore, the sendersTo relation can be reconfigured at any time based on the communication patterns of the application.

\section{B. Optimistic total order}

Optimistic techniques have been used before to reduce the latency of atomic broadcast. To our knowledge, the protocol proposed in this paper is the first to exploit optimism in atomic multicast. Moreover, differently from previous approaches, our optimistic assumption does not rely on spontaneous total order, a property that typically holds in local-area networks under moderate load, but not necessarily in geographically distributed systems.

Two optimistic atomic broadcast protocols are proposed in [5] and [7]. In both cases, if the optimistic assumption holds, messages are delivered within two communication delays. The idea of the algorithm in [5] is to rely on spontaneous total order to avoid ordering through consensus messages that have already been spontaneously ordered by the network. Fast Paxos [7] is an extension of the classic Paxos algorithm. Differently from classic Paxos, messages are sent directly to acceptors (skipping the leader). If messages are received in the same order, delivery is done in two communication steps; otherwise, it takes longer.

Broadcast algorithms that take into consideration application semantics to deliver messages fast have been also proposed [8], [9], [10]. To a certain extent, these algorithms "optimistically" assume that not every two messages must be delivered in the same order by all processes, but only some messages (e.g., messages that access shared objects). Optimistic Atomic Multicast does not rely on application semantics. We note however that application semantics could be used when deciding whether the order of optimistic and conservative delivery match.

In [6], the authors propose a technique that approximates spontaneous ordering in a wide-area setting. The key idea is for every process to insert artificial delays in incoming messages. The resulting delay between each process $p$ and every other process should then become the same as the delay between $p$ and a given sequencer $s$ (e.g., the leader in a consensus-based protocol). For the technique to work properly, the communication latency between each pair of processes has to be approximately constant. Our optimistic delivery does not have one single sequencer process as a reference, as opposed to [6]. Instead, given a set of processes which communicate with one another, each one of such processes is able to determine an optimistic ordering based solely on its own wait window and on messages' timestamps. Furthermore, the authors propose an atomic 
broadcast protocol, ${ }^{4}$ where the latency between every single pair or processes must be constant, whereas we propose an optimistic atomic multicast protocol which cares only about those pairs of processes that can communicate, as defined by the sendersTo relation.

\section{CONCLUSION}

This work introduced the concept of quasi-genuine multicast, a class of protocols based on pre-determined communication patterns (i.e., the sendersTo relation). Such patterns are based on the application and may be changed on-the-fly (i.e., by means of a system reconfiguration). Experimental results in both local-area and wide-area networks show that Optimistic Atomic Multicast is effective in increasing throughput and reducing latency. Low latency is due to a probabilistic assumption that was verified in $95 \%$ of deliveries in a wide-area network and over $99.5 \%$ in a localarea network. As a future work, we intend to investigate protocols that automatically detect communication patterns and reconfigure the system as pairs of groups communicate more or less frequently.

\section{REFERENCES}

[1] L. Lamport, "Time, clocks, and the ordering of events in a distributed system," Communications of the ACM, vol. 21, no. 7, pp. 558-565, 1978.

[2] F. B. Schneider, "What good are models and what models are good?," in Distributed Systems (S. Mullender, ed.), ch. 2, Addison-Wesley, 2nd ed., 1993.

[3] V. Hadzilacos and S. Toueg, Fault-tolerant broadcasts and related problems, pp. 97-145. New York, NY, USA: ACM Press/Addison-Wesley Publishing Co., 1993.

[4] L. Lamport, "Lower bounds for asynchronous consensus," Distributed Computing, vol. 19, no. 2, pp. 104-125, 2006.

[5] F. Pedone and A. Schiper, "Optimistic atomic broadcast: a pragmatic viewpoint," Theoretical Computer Science, vol. 291 , no. 1 , pp. $79-101,2003$.

[6] A. Sousa, J. Pereira, F. Moura, and R. Oliveira, "Optimistic total order in wide area networks," in Proceedings of the 21st IEEE Symposium on Reliable Distributed Systems, SRDS '02, pp. 190-199, IEEE Computer Society, 2002.

[7] L. Lamport, "Fast paxos," Distributed Computing, vol. 19, no. 2, pp. 79-103, 2006.

[8] F. Pedone and A. Schiper, "Generic broadcast," in Proceedings of the 13th International Symposium on Distributed Computing (DISC'99, formerly WDAG), 1999.

[9] L. Lamport, "Generalized consensus and paxos," Tech. Rep. MSR-TR-2005-33, Microsoft Research (MSR), Mar. 2005.

\footnotetext{
${ }^{4}$ In [6], the protocol is called "atomic multicast", but is defined as a protocol in which every correct process delivers all messages multicast by all correct processes. From our definitions, we call it "atomic broadcast".
}

[10] P. Sutra and M. Shapiro, "Fast Genuine Generalized Consensus," in Symposium on Reliable Distributed Systems, (Madrid, Spain), Oct. 2011.

[11] R. Guerraoui and A. Schiper, "Genuine atomic multicast in asynchronous systems,” Tech. Rep. 98/273, EPFL, Mar. 1998.

[12] C. Curino, E. Jones, Y. Zhang, and S. Madden, "Schism: a workload-driven approach to database replication and partitioning," Proc. VLDB Endow., vol. 3, pp. 48-57, 2010.

[13] C. E. Bezerra, F. Pedone, B. Garbinato, and C. Geyer, "Optimistic atomic multicast," Tech. Rep. 2013/01, University of Lugano, Feb. 2013.

[14] N. Schiper and F. Pedone, "On the inherent cost of atomic broadcast and multicast in wide area networks," in Proceedings of the 9th international conference on Distributed computing and networking, ICDCN'08, (Berlin, Heidelberg), pp. 147-157, 2008.

[15] B. Kemme, F. Pedone, G. Alonso, A. Schiper, and M. Wiesmann, "Using optimistic atomic broadcast in transaction processing systems," IEEE Trans. Knowl. Data Eng., vol. 15, no. 4, pp. 1018-1032, 2003.

[16] F. R. Cecin, C. F. R. Geyer, S. Rabello, and J. L. V. Barbosa, "A peer-to-peer simulation technique for instanced massively multiplayer games," in Proceedings of the 10th IEEE international symposium on Distributed Simulation and Real-Time Applications, pp. 43-50, 2006.

[17] L. Lamport, "The part-time parliament," ACM Transactions on Computer Systems (TOCS), vol. 16, no. 2, pp. 133-169, 1998.

[18] X. Défago, A. Schiper, and P. Urbán, "Total order broadcast and multicast algorithms: Taxonomy and survey," ACM Comput. Surv., vol. 36, pp. 372-421, December 2004.

[19] M. K. Aguilera and R. E. Strom, "Efficient atomic broadcast using deterministic merge," in Proceedings of the nineteenth annual ACM symposium on Principles of distributed computing, (New York, NY, USA), pp. 209-218, ACM, 2000.

[20] K. Birman and T. Joseph, "Reliable communication in the presence of failures," ACM Transactions on Computer Systems (TOCS), vol. 5, no. 1, pp. 47-76, 1987.

[21] L. Rodrigues, R. Guerraoui, and A. Schiper, "Scalable atomic multicast," in Computer Communications and Networks, 1998. Proceedings. 7th International Conference on, pp. 840 -847 , oct 1998.

[22] J. Fritzke, U., P. Ingels, A. Mostefaoui, and M. Raynal, "Faulttolerant total order multicast to asynchronous groups," in Reliable Distributed Systems, 1998. Proceedings. Seventeenth IEEE Symposium on, pp. 228-234, 1998. 